\title{
Perinatal Hypophosphatasia in a Premature Infant
}

\author{
Deepika Sankaran, MD ${ }^{1}$ Praveen K. Chandrasekharan, MD, MS² Munmun Rawat, MD² \\ 1 Department of Pediatrics, University of California, Davis, \\ Sacramento, California \\ 2 Department of Pediatrics, University at Buffalo, Buffalo, New York \\ Address for correspondence Deepika Sankaran, MD, Division of \\ Neonatology, Department of Pediatrics, University of California, \\ 2516 Stockton Boulevard, Davis, Sacramento, CA 95817 \\ (e-mail: dsankaran@ucdavis.edu; deepikasnkrn@gmail.com).
}

Am J Perinatol Rep 2020;10:e139-e147.

\begin{abstract}
Keywords

- hypophosphatasia

- ossification

- alkaline phosphatase

A premature male infant was delivered at 32 weeks' gestation due to category-2 fetal tracing after preterm labor. The physical exam showed shortened and bowed long bones, with calvarium felt in small area of the head. Serum alkaline phosphatase was very low on admission. Extensive metaphyseal abnormalities, bowing of long bones, and poor ossification of all bones were noted on skeletal survey (radiography). Based on ultrasound evidence of "bowing" and long bone fractures at 26 weeks, amniocentesis was performed that later diagnosed hypophosphatasia by genetic testing while ruling out osteogenesis imperfecta. Although ventilated initially, the infant gradually improved with enzyme replacement therapy (ERT) and was extubated to noninvasive ventilation for 6 weeks. Following clinical deterioration with hypoxic respiratory failure secondary to sepsis at 4 months of age, he succumbed to severe pulmonary hypertension, likely secondary to chronic lung disease and prolonged ventilation. Early diagnosis allowed timely initiation of appropriate therapy. Radiological improvement with the therapy showed promising results in this rare disease. Despite specific novel therapy being available, variability in presentation dictates prognosis in this previously universally fatal condition. The potential unknown effects of ERT on pulmonary vascular remodeling need further investigation.
\end{abstract}

Hypophosphatasia (HPP) is an inherited disorder characterized by defective bone mineralization and low alkaline phosphatase (ALP) activity. The clinical spectrum is extremely variable, ranging from very severe perinatal form to a mild form with late onset, presenting in adults with nonspecific arthropathy, musculoskeletal pain, and premature exfoliation of teeth., ${ }^{1,2}$ Prior to availability of enzyme replacement therapy (ERT), perinatal severe HPP was lethal in all cases. ERT with asfotase alfa is currently the only approved treatment for HPP.

Despite the rarity of this condition, early identification by antenatal imaging was pivotal to manage this infant appropriately with the ERT. Secondary to prematurity with chronic lung disease in addition to the risk of pulmonary hypoplasia, the infant was predisposed to developing complications such as pulmonary hypertension.

received

December 16, 2019

accepted

March 5, 2020
DOI https://doi.org/

10.1055/s-0040-1709512. ISSN 2157-6998.

\section{Case Presentation}

The maternal care of a 28-year-old gravida 5, African American female with three prior healthy children was transferred to Regional Perinatal Center (RPC) for suspected osteogenesis imperfecta (OI) versus HPP in the fetus based on antenatal imaging. Ultrasound performed at 26 weeks showed abnormalities of bone mineralization, length and shape with possible fractures consistent with OI or other skeletal dysplasia; all long bones were noted to be short and bowed, with all measurements below first percentile. The estimated fetal weight was at 27 th percentile. Amniocentesis was performed and the results were pending at the time of birth. Maternal prenatal laboratory results were negative except for positive vaginal group B streptococcal culture. Family history was negative for inherited disorders.

Copyright $\odot 2020$ by Thieme Medical Publishers, Inc., 333 Seventh Avenue, New York, NY 10001, USA. Tel: +1(212) 760-0888.
License terms

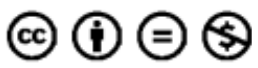


Mother was admitted to our RPC due to preterm labor with premature rupture of membranes for 12 days and received betamethasone for fetal lung maturity. Due to category-2 fetal heart tracing with placental abruption, an emergency classical cesarean section was performed under general anesthesia to deliver a male infant at $32^{2 / 7}$ weeks' gestation. In the delivery room, the infant required face mask continuous positive airway pressure due to respiratory distress, and subsequently, he was placed on noninvasive positive pressure ventilation (NIPPV) on admission to neonatal intensive care unit. His birth weight was appropriate for gestational age and his physical exam on admission was significant for shortening and bowing of all four extremities that was more prominent in upper than in lower extremities ( - Fig. 1); the calvarium was felt only over a small area of frontal aspect of the head and the rest of his scalp had soft consistency. A skeletal survey (-Figs. 2-4) was performed that showed extensive metaphyseal abnormalities and bowing of long bones with poor ossification of all bones suggestive of HPP.

The infant's respiratory distress was most likely secondary to retained fetal lung fluid as confirmed with the chest radiograph ( - Fig. 5A), which also showed well-inflated lungs, thus ruling out respiratory distress syndrome and pulmonary hypoplasia. He was continued on NIPPV via ram cannula with fraction of inspired oxygen $\left(\mathrm{FiO}_{2}\right)$ of 0.21 to 0.25 . He also had transient hypoglycemia, likely due to prematurity, that was managed with $10 \%$ dextrose boluses and total parenteral nutrition (TPN) via an umbilical venous catheter. On day 2 of postnatal life, the genetic testing from amniocentesis confirmed the diagnosis as HPP; two heterozygous pathogenic variants in ALPL gene were identified, with variants residing on different alleles consistent with a recessive form of HPP (first variant: c.1348C $>$ T, p.Arg450Cys and second variant: c.1375G > A, p.Val459Met).

His serum ALP level on admission was $8 \mathrm{U} / \mathrm{L}$ that supported the diagnosis of perinatal severe HPP. The serum calcium and vitamin $D$ levels were high and were restricted in the TPN to avoid toxicity. On day 3 of postnatal life, he was started on enteral feeds via an orogastric tube and a peripherally inserted central catheter (PICC) was placed. He received 48 hours of antibiotics due to suspected early-onset sepsis, which was ruled out based on negative initial blood culture. Due to intolerance to bolus feeds, he was started on continuous feeds that were advanced gradually until full feeds were achieved by 1 month of age and his PICC was discontinued. The pediatric endocrinologist was consulted, who recommended gentle handling, testing for vitamin B6 level, while the neurosurgery team recommended immobilization of head and spine. The metabolic/genetics subspecialist along with the endocrinologist recommended initiation of ERT. On day 5 of postnatal life, he was started on ERT with asfotase alfa (Strensiq; Alexion pharmaceuticals, Boston, MA) $2 \mathrm{mg} / \mathrm{kg}$ subcutaneous injection three times a week, along with close monitoring of serum calcium and ALP levels. The pulmonologist was consulted due to the risk of pulmonary hypoplasia secondary to small chest size that has been previously described ${ }^{3}$ and they guided the respiratory management.

\section{Investigations}

Skeletal survey (Figs. 2-4) was performed at birth that demonstrated extensive metaphyseal abnormalities, multiple rib fractures, and bowing of long bones with poor ossification of all bones. Serum ALP level at birth was very low at $8 \mathrm{U} / \mathrm{L}$ (normal range: $150-420 \mathrm{U} / \mathrm{L}$ ) and his serum calcium level of $12.1 \mathrm{mg} / \mathrm{dL}$ (normal range: $7.6-10.4 \mathrm{mg} / \mathrm{dL}$ ) with ionized calcium of $7.1 \mathrm{mg} / \mathrm{dL}$ (normal range: 3.9-6.0 $\mathrm{mg} / \mathrm{dL}$ ) was very high. The vitamin $\mathrm{B} 6$ level was very high at $1,450 \mu \mathrm{g} / \mathrm{L}$ (normal $<46$ ) and 25-hydroxy vitamin $\mathrm{D}$ was low at $12 \mathrm{ng} / \mathrm{mL}$ (normal >30) (-Table 1). Urinalysis showed increased calcium/creatinine ratio (1.04, normal range is $<0.7$ ). Computed tomography (CT) scan of the cervical, thoracic, and lumbar spine showed persistent heterogeneous ossification with interval increased ossification compared with the skeletal survey. Vertebral bodies showed mild dextroconvex curvature in the thoracolumbar region.

\section{Differential Diagnosis}

The major differential diagnoses of perinatal severe HPP based on antenatal imaging include OI types 2 and 3 (type 2 is the perinatal lethal form and type 3 is the severe form that affects surviving neonates). The other conditions that could have a similar clinical picture include campomelic dysplasia, chondrodysplasia with defective mineralization, X-linked hypophosphatemia, and achondrogenesis. ${ }^{4}$ Physical exam findings are very similar between perinatal severe HPP and OI. Perinatal HPP is the only condition with very low serum ALP which is pathognomic along with high serum pyridoxal phosphate or pyridoxal $5^{\prime}$ phosphate (PLP) (-Table 2).

\section{Treatment}

A multidisciplinary team including neonatologists, pulmonologists, endocrinologists, metabolic/geneticists, cardiologists, and neurosurgeons was involved in the management of this infant. The infant was managed with supportive treatment including nutritional support with enteral feeds and TPN. His pain was managed with Tylenol and morphine as needed. His respiratory insufficiency was managed with prolonged ventilation-both invasive and NIPPV. He was placed on a custom-made whole-body brace by the neurosurgery team for immobilization to avoid brain and spinal cord injury. On postnatal day 5 , he was started on ERT.

Gradually, he had an increase in serum ALP levels, with mineralization of bones over the next 6 to 8 weeks ( - Fig. 5A, B), along with a decrease in serum total and ionized calcium (-Fig.6A, B). Adverse effects that have been reported include increased risk of fractures, craniosynostosis, and local reactions at the site of injection that could be severe with hypersensitivity, lipodystrophy, and ectopic calcifications that can be avoided by rotating the site; he did not have any adverse effects locally in the injection site. ${ }^{5}$ 


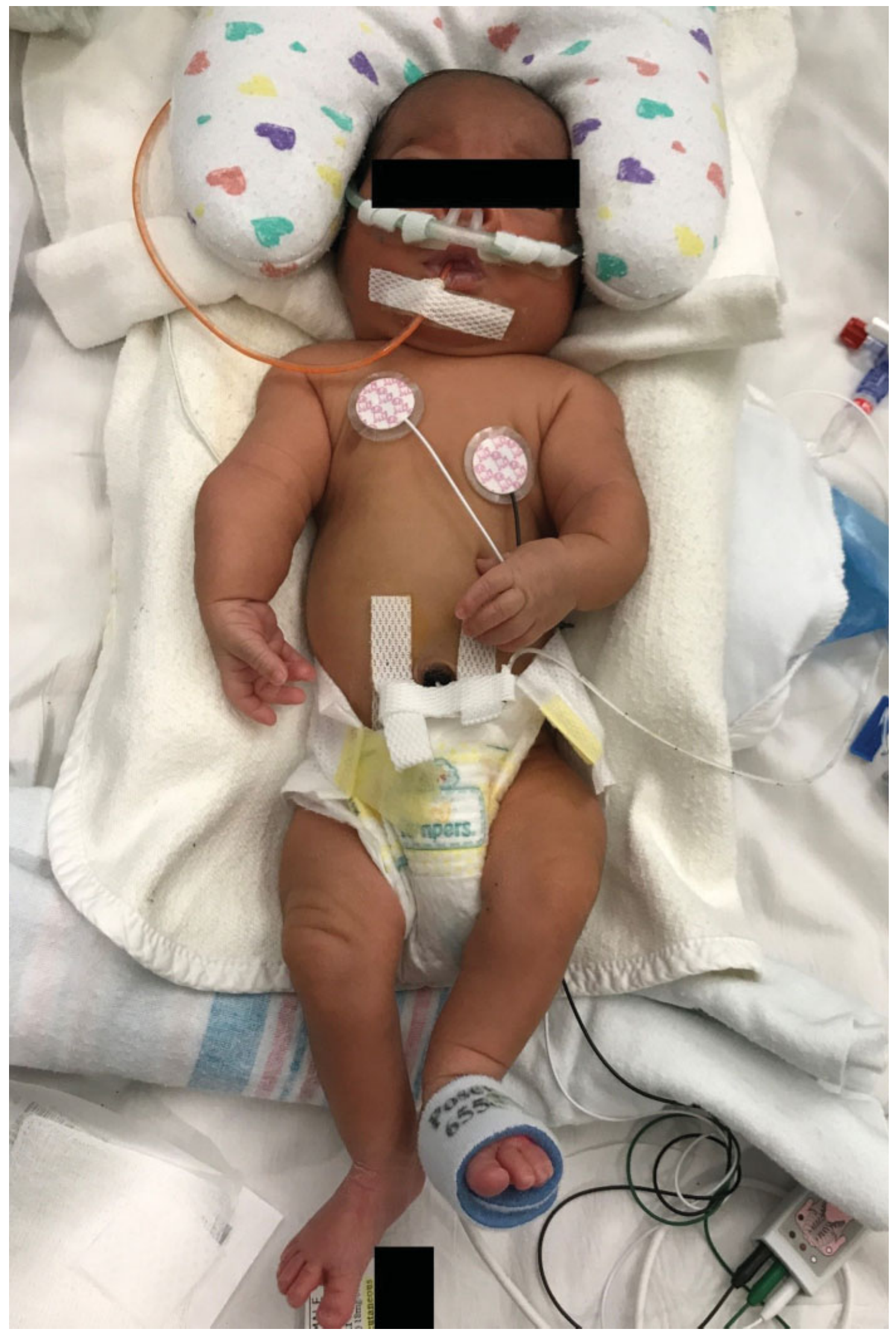

Fig. 1 Infant at 5 days after birth. Shortening and bowing of extremities noted. 

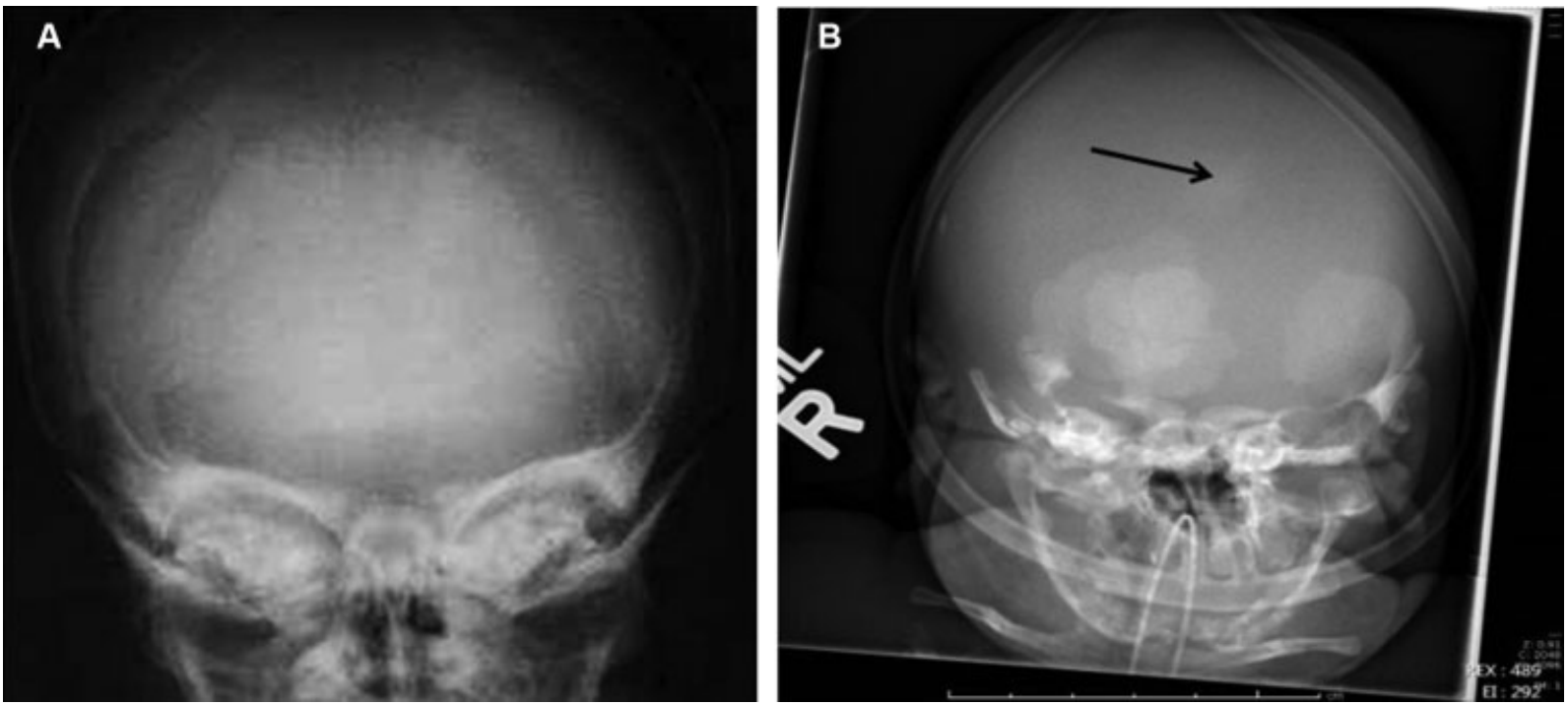

Fig. 2 Anteroposterior plain skull radiograph of infant (B), compared with a normal newborn infant's skull radiograph (A): generalized poor ossification of skull noted in infant with hypophosphatasia (black arrow).
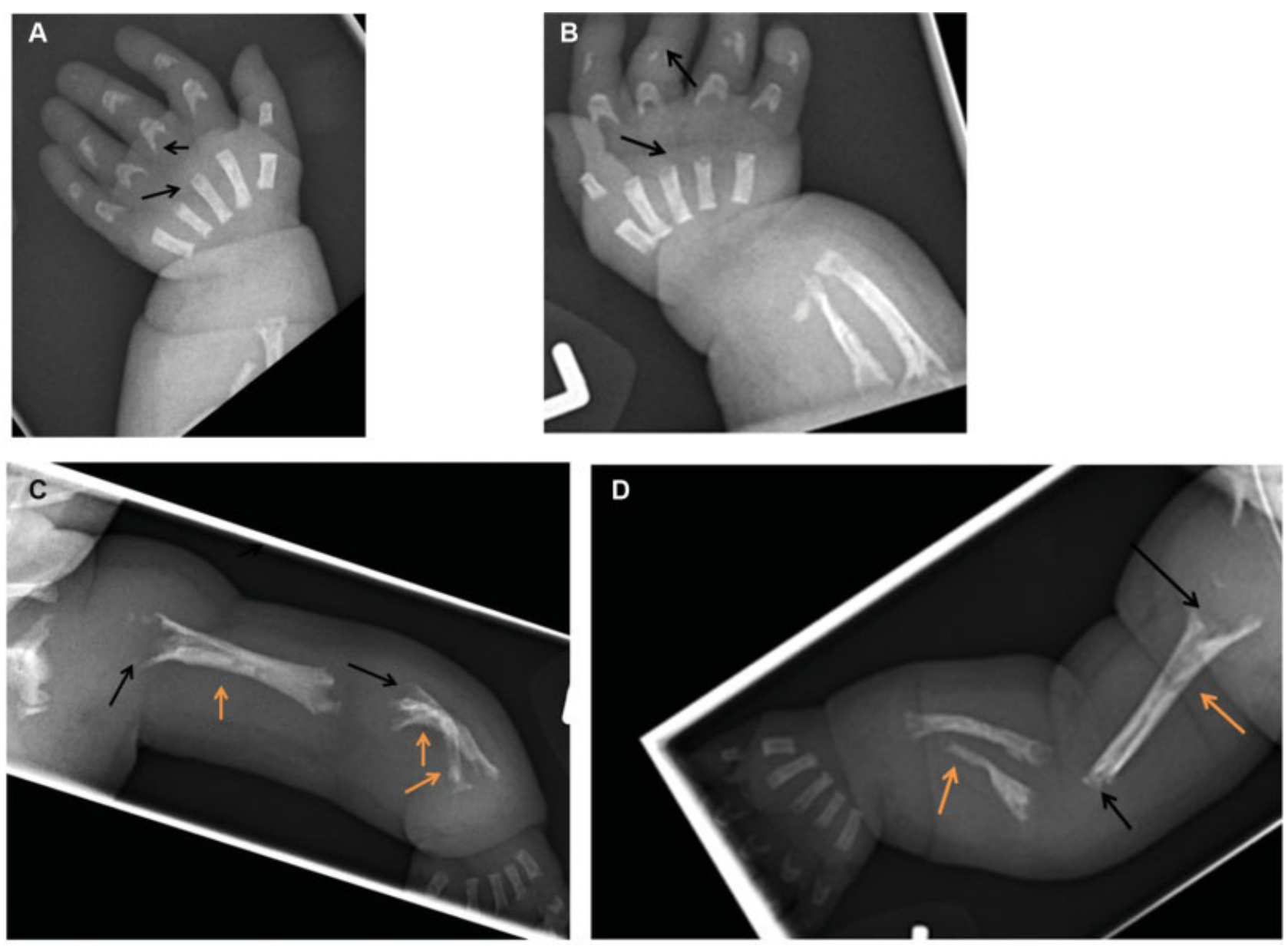

Fig. 3 Plain radiography of upper extremities: (A, B) anteroposterior view of the left and the right hands with part of the forearm demonstrate abnormal lucencies within the proximal phalanges and lack of ossification in the distal phalanges. (C, D) Anteroposterior view of the left and the right arms and forearms show similar findings of poor ossification in both the metaphyses (black arrows) and diaphyses (orange arrows) of all the long bones in the bilateral upper extremities with bowing and shortening. 

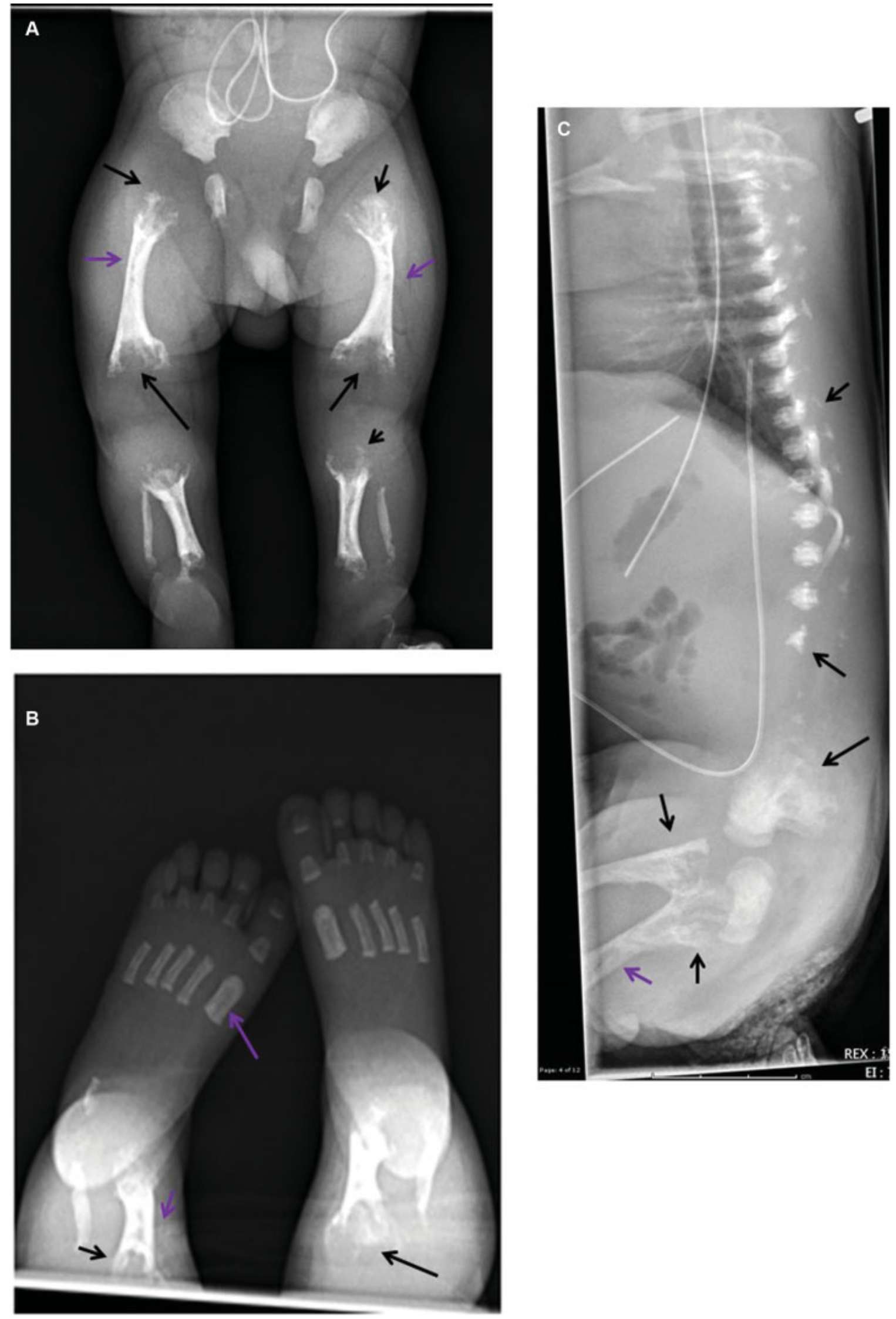

Fig. 4 Plain radiography of lower extremities and spine: extensive metaphyseal abnormality of all the long bones, bowing and shortening of femur, tibia, and fibula (A); irregular lucency and possible destructive process of metaphyses (black arrows) and diaphysis (purple arrows) of all bones including metatarsals (B), poor ossification of cervical, lower lumbar and sacral spines, dysplastic upper lumbar and thoracic vertebral bodies (C). 

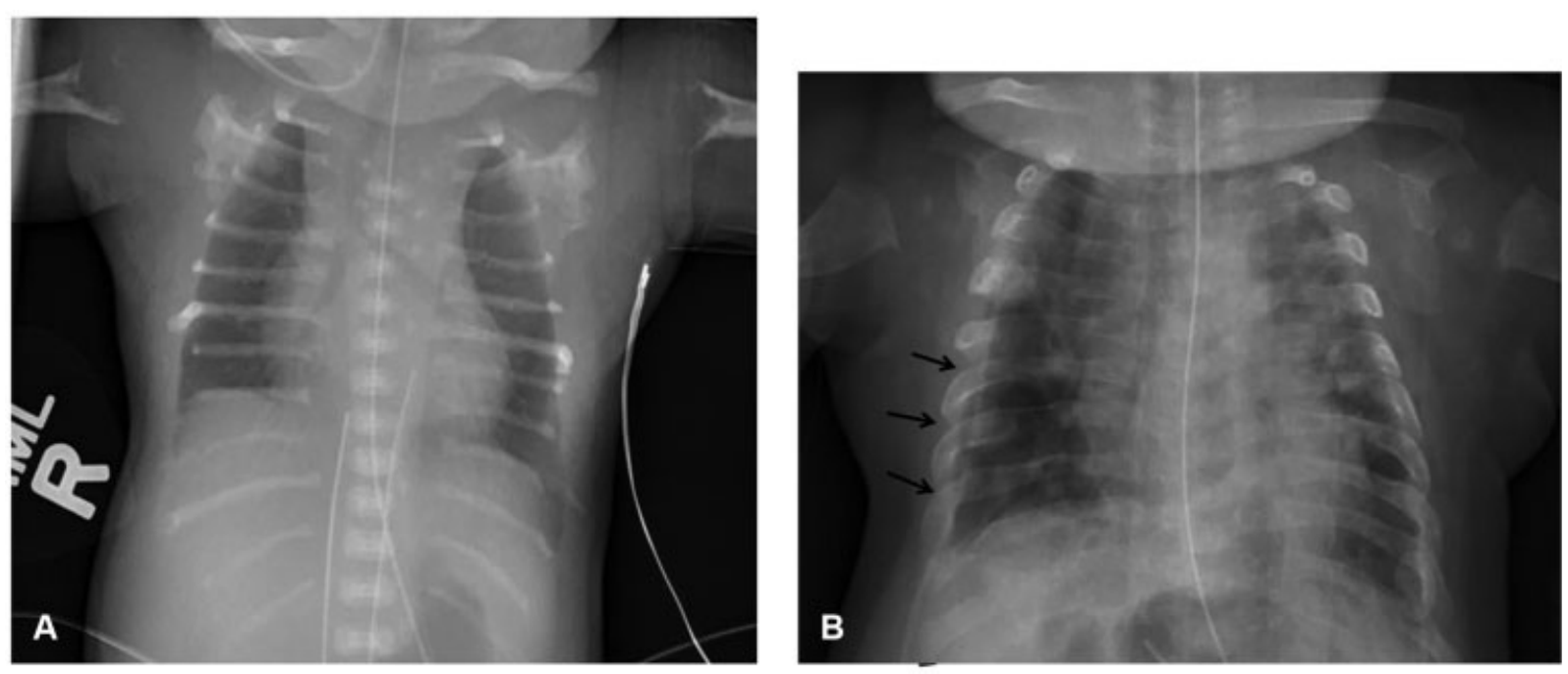

Fig. 5 Improvement in mineralization noted with enzyme replacement therapy: comparison of chest radiograph at birth (A) and at 3 months (B). Black arrows demonstrate the improved ossification.

Table 1 Pertinent laboratory investigations in the infant

\begin{tabular}{|l|l|l|}
\hline $\begin{array}{l}\text { Laboratory test } \\
\text { in Serum }\end{array}$ & $\begin{array}{l}\text { Result in } \\
\text { patient }\end{array}$ & Normal range $^{\mathbf{a}}$ \\
\hline Alkaline phosphatase & $8 \mathrm{U} / \mathrm{L}$ & $150-420 \mathrm{U} / \mathrm{L}$ \\
\hline Total calcium & $12.1 \mathrm{mg} / \mathrm{dL}$ & $8.5-10.5 \mathrm{mg} / \mathrm{dL}$ \\
\hline Ionized calcium & $7.1 \mathrm{mg} / \mathrm{dL}$ & $4.4-5.4 \mathrm{mg} / \mathrm{dL}$ \\
\hline Vitamin B6 & $1,450 \mu \mathrm{g} / \mathrm{L}$ & $<46 \mu \mathrm{g} / \mathrm{L}$ \\
\hline 25-hydroxy vitamin D & $12 \mathrm{ng} / \mathrm{mL}$ & $>30 \mathrm{ng} / \mathrm{mL}$ \\
\hline
\end{tabular}

${ }^{\text {a }}$ Reference ranges from the book: The Harriet Lane Handbook, 19th edition.

\section{Outcome and Follow-up}

The infant tolerated ERT well and the dose was increased to $3 \mathrm{mg} / \mathrm{kg}$ on day 10 of postnatal life; calcium carbonate supplements were started at 1 month of life due to a gradual decline in the serum total calcium levels. He achieved full enteral feeds and PICC was discontinued by 1 month. At 6 weeks of age, he self-extubated and was placed on NIPPV. He had limited spontaneous movements of his limbs, possibly secondary to pain and from being immobilized with a custom-made brace. Serial chest radiographs (-Fig. 5A, 5B) and noncontrast CT scan of the head both showed evidence of improved mineralization. He was stable on noninvasive ventilation requiring minimal support with $\mathrm{FiO}_{2}$ of 0.21 to 0.30 until day 118 when he had a clinical decompensation with respiratory failure and suspected sepsis. He was intubated and placed on high-frequency oscillator with high mean airway pressure and $\mathrm{FiO}_{2}$ of 1 to achieve adequate lung inflation and preductal oxygen saturation of $>95 \%$. His tracheal culture was positive for Klebsiella pneumoniae that was managed with appropriate antibiotics. He did not have an echocardiogram performed during the first 3 months after birth until he deteriorated clinically due to sepsis. During his acute clinical worsening, his transthoracic echocardiogram demonstrated suprasystemic pulmonary hypertension (and structurally normal heart with no patent ductus arteriosus) that remained refractory. He did not improve despite maximal support with pulmonary vasodilators (nitric oxide and milrinone), inotropes inotrope (dopamine), vasopressor (vasopressin), and sedation (fentanyl, midazolam, and dexmedetomidine). After extensive discussion involving a multidisciplinary team including pediatric critical care, pulmonology, and genetic/metabolic subspecialists, the parents chose the option of comfort care and his life support was withdrawn as desired by the

Table 2 Laboratory evaluation to differentiate hypophosphatasia from X-linked hypophosphatemic rickets and osteogenesis imperfecta in a neonate 2,25

\begin{tabular}{|l|l|l|l|}
\hline Parameter (sample: serum) & Hypophosphatasia & X-linked hypophosphatemic rickets & Osteogenesis imperfecta \\
\hline Alkaline phosphatase & Decreased & Increased & Normal \\
\hline Pyridoxal 5' phosphate & Increased & Decreased & - \\
\hline Calcium & Increased/normal & Normal & Normal \\
\hline Phosphate & Increased/normal & Decreased & Normal \\
\hline Parathyroid hormone & Decreased/normal & Normal & Normal \\
\hline Vitamin D & Normal & Decreased/normal & Normal \\
\hline
\end{tabular}



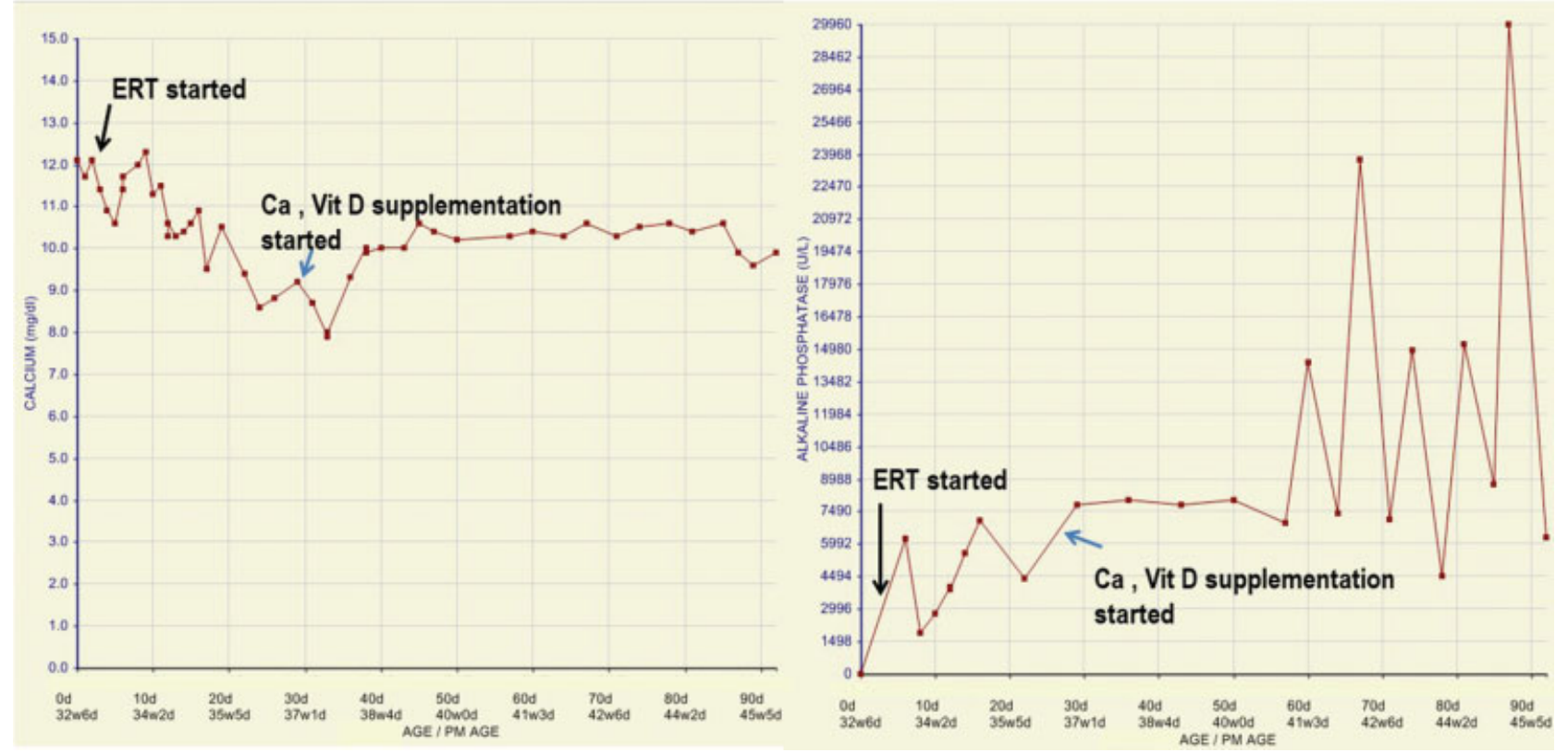

Fig. 6 Changes in serum calcium and ALP levels: Following initiation of ERT, the calcium level that was initially high gradually decreased to normal levels; when it dropped further lower, calcium and vitamin D supplementation was started (A). Soon after ERT was initiated, the ALP levels increased to several thousands, stabilized at $\sim 7,500 \mathrm{U} / \mathrm{L}(\mathrm{B})$ and subsequently had intermittent "peaks" in the levels while on ERT. ALP, alkaline phosphatase; ERT, enzyme replacement therapy. Ca, Total serum calcium; VitD, 25-hydroxy Vitamin D.

parents on day 128 of postnatal age. The parents declined an autopsy.

\section{Discussion}

HPP is a rare inborn error of metabolism that was first described in the literature by Rathbun. ${ }^{6}$ HPP is caused by a loss of function mutation of ALPL gene that encodes tissuenonspecific alkaline phosphatase (TNSALP) isoenzyme, a cell surface protein that is expressed highly in liver, bone, and kidney, ${ }^{7}$ but also in teeth, central nervous system, endothelial cells, and fibroblasts. ${ }^{8}$ The substrates of TNSALP, including inorganic pyrophosphate (a potent inhibitor of mineralization), accumulate extracellularly in HPP resulting in systemic and metabolic disease. The clinical presentation is extremely variable, ranging from very severe, mostly lethal perinatal form to a mild form with late/adult onset presenting with arthropathy and musculoskeletal pain. ${ }^{1,2}$ The classification of HPP that is used currently is based on age at diagnosis and presence of bone symptoms; HPP is classified into six types: perinatal, prenatal benign, infantile, childhood, adult, and odontohypophosphatasia. Odontohypophosphatasia is the least severe form of HPP, with premature exfoliation of primary and/or permanent teeth and/or severe dental caries, in the absence of skeletal system abnormalities. ${ }^{9}$

Perinatal severe HPP is the most severe form, inherited as autosomal recessive, and was lethal in all cases prior to the availability of ERT. The affected infants would pass away at birth or in the first few days of life from respiratory insufficiency due to chest deformities and lung hypoplasia. These infants also developed apnea and vitamin B6-dependent epileptic seizures due to disruption in the levels of PLP, a substrate of TNSALP in neuronal cells resulting in central nervous system localized vitamin B6 deficiency. ${ }^{10-12}$ Perinatal HPP is the only condition with very low serum ALP that is pathognomic along with high serum PLP (PLP is hydrolyzed by TNSALP expressed in brain; unphosphorylated pyridoxal crosses blood-brain barrier to be regenerated as PLP in the cell, which acts as a coenzyme for glutaric acid decarboxylase needed for synthesis of gamma-amino butyric acid, a neurotransmitter). It can be antenatally diagnosed using ultrasound: evidence of poor mineralization, bowed or shorter long bones, fractures and specific signs such as osteochondral spurs (Bowdler spurs), ${ }^{13}$ and pretibial dimpling. ${ }^{14}$ These specific signs are absent in the prenatal benign form ("bent but not broken") in which the pathological signs regress gradually from the third trimester. ${ }^{15}$ Extreme skeletal disease is obvious at birth with short bowed bones, poor or no mineralization resulting in pathological fractures (metaphyseal fractures), deformities with short and bowed bones, thoracic instability, and pulmonary hypoplasia. The classic findings on radiographs include extensive metaphyseal abnormalities with poor mineralization, shortened and bowed long bones, and generalized lack of ossification. The infants are at risk of respiratory insufficiency and a need for prolonged ventilation ${ }^{16}$; they are also at risk of sustaining brain and spinal cord injury from poor mineralization of the skull and spine. Affected infants can have hypercalcemia, hypercalciuria, nephrocalcinosis, and ophthalmic calcification from calcium deposits. ${ }^{17,18}$

The hallmark of perinatal severe HPP is low ALP levels; more severe the disease, lower the ALP activity. The other characteristic laboratory findings include high serum PLP (the active form of vitamin B6 that requires ALP as a cofactor for it to be broken down into vitamin B6), high urinary phenylethanolamine (which is also a substrate for TNSALP) and high urinary inorganic pyrophosphate. High serum PLP is a sensitive marker 
that correlates with severity. The serum calcium level is initially high prior to treatment, whereas they have normal serum levels of 25-hydroxy vitamin D, parathyroid hormone, and phosphorus.

The genetic testing by mutation screening with sequencing of the ALPL coding sequence and the intron/exon borders is essential to confirm the diagnosis and to offer a molecular prenatal diagnosis to help with genetic counseling of families affected by this condition. In the premature infant described here, the infant had the pathognomonic signs on clinical exam and laboratory investigations at presentation (after birth) and that combined with the availability of prenatal confirmatory testing from amniocentesis allowed definitive diagnosis and hence timely initiation of ERT. In the absence of genetic testing (or while waiting for the results), clinical presentation along with very low ALP will help in diagnosing perinatal severe HPP, differentiating it from OI. The management includes supportive treatment and ERT. The supportive treatment comprises analgesics and need for sedation to manage the pain, nutritional management with parenteral and enteral nutrition with close monitoring of calcium and phosphorous, to support optimal growth. The management of respiratory insufficiency may involve prolonged ventilation for 6 to 12 months, with potential need for tracheostomy. Tracheostomy should be offered to the patients with perinatal HPP, who require significant respiratory support during hospitalization to assist with continued support and to facilitate their discharge to home/long-term care facility. Vitamin B6 therapy and antiepileptics may be needed in the presence of seizures. Spine stabilization with a brace is recommended to avoid spinal cord injury, the duration of which would depend on the amount of ossification achieved with the use of ERT that can be evaluated with follow-up imaging, with not much evidence available due to very few survivors with perinatal HPP. An eye exam by ophthalmology is required to look for signs of raised intracranial pressure and calcium deposition.

There have been several previously reported cases of perinatal severe HPP, but we report one of the few in premature infants. ${ }^{16,19,20}$

ERT with asfotase alfa (Strensiq) has shown promising results in this debilitating disease. ${ }^{21}$ Asfotase alfa is a recombinant human bone targeted TNSALP. It has been the U.S. Food and Drug Administration approved for perinatal, infantile, and childhood HPP since 2015. It has been previously reported that ERT results in increased osteoid volume and thickened bones based on bone biopsy. ${ }^{22}$ Improved survival has been noted in treated infants: 95 versus $42 \%$ by 1 year of age and 84 versus $27 \%$ by 5 years of age. ${ }^{23}$ Better respiratory outcomes with the use of ERT have been shown by Whyte et al: 76\% (16/21) of the ventilated and treated patients survived among whom $75 \%$ $(12 / 16)$ were weaned from ventilatory support after treatment as compared with only $5 \%$ (1/20) of historical controls needing ventilatory support who survived. ${ }^{23}$ A recent open-label multinational study to determine the long-term safety and efficacy of asfotase alfa showed that most patients improved radiographically and clinically in the first 6 years, while radiographic nonresponders had worse disease at baseline and greater amounts of neutralizing antibodies. ${ }^{5}$ A multidisciplinary approach involving pediatric endocrinology, metabolic/genetics team, pulmonology, neurosurgery, pain management, and ophthalmology is warranted for the optimal care of such patients. $^{24}$

\section{Conclusion}

In this particular patient, it is unclear if the rapid decompensation could have been due to gradual development of respiratory insufficiency secondary to restrictive lung disease (possibly worsened by increased ossification) with tilt in the balance due to sepsis. The effect of the novel therapy (ERT) on pulmonary vascular remodeling is unknown and needs further investigation. Since cardiac catheterization was not performed for this infant and the parents did not consent for an autopsy, we do not have answers with respect to the condition of the pulmonary vasculature and histology of the lungs, respectively. Reporting of similar cases would be extremely beneficial for the future reference and aid in the appropriate approach to infants with perinatal severe HPP.

\section{Conflict of Interest \\ None declared.}

\section{Acknowledgment}

We acknowledge the parents of the infant who gave consent for this case report.

\section{References}

1 Whyte MP, Wenkert D, Zhang F. Hypophosphatasia: natural history study of 101 affected children investigated at one research center. Bone 2016;93:125-138

2 Whyte MP. Hypophosphatasia and the role of alkaline phosphatase in skeletal mineralization. Endocr Rev 1994;15(04):439-461

3 Silver MM, Vilos GA, Milne KJ. Pulmonary hypoplasia in neonatal hypophosphatasia. Pediatr Pathol 1988;8(05):483-493

4 Offiah AC, Vockley J, Munns CF, Murotsuki J. Differential diagnosis of perinatal hypophosphatasia: radiologic perspectives. Pediatr Radiol 2019;49(01):3-22

5 Hofmann CE, Harmatz P, Vockley J, et al; ENB-010-10 Study Group. Efficacy and safety of asfotase alfa in infants and young children with hypophosphatasia: a phase 2 open-label study. J Clin Endocrinol Metab 2019;104(07):2735-2747

6 Rathbun JC. Hypophosphatasia; a new developmental anomaly. Am J Dis Child 1948;75(06):822-831

7 Weiss MJ, Cole DE, Ray K, et al. A missense mutation in the human liver/bone/kidney alkaline phosphatase gene causing a lethal form of hypophosphatasia. Proc Natl Acad Sci U S A 1988;85 (20):7666-7669

8 Mornet E. Hypophosphatasia. Metabolism 2018;82:142-155

9 Mornet E. Hypophosphatasia. Orphanet J Rare Dis 2007;2:40-40

10 Baumgartner-Sigl S, Haberlandt E, Mumm S, et al. Pyridoxineresponsive seizures as the first symptom of infantile hypophosphatasia caused by two novel missense mutations (c.677T $>C$, p. M226T; c.1112C > T, p.T371I) of the tissue-nonspecific alkaline phosphatase gene. Bone 2007;40(06):1655-1661

11 Nunes ML, Mugnol F, Bica I, Fiori RM. Pyridoxine-dependent seizures associated with hypophosphatasia in a newborn. J Child Neurol 2002;17(03):222-224

12 Demirbilek H, Alanay Y, Alikaşifoğlu A, et al. Hypophosphatasia presenting with pyridoxine-responsive seizures, hypercalcemia, 
and pseudotumor cerebri: case report. J Clin Res Pediatr Endocrinol 2012;4(01):34-38

13 Shohat M, Rimoin DL, Gruber HE, Lachman RS. Perinatal lethal hypophosphatasia; clinical, radiologic and morphologic findings. Pediatr Radiol 1991;21(06):421-427

14 Zankl A, Mornet E, Wong S. Specific ultrasonographic features of perinatal lethal hypophosphatasia. Am J Med Genet A 2008;146A (09):1200-1204

15 Pauli RM, Modaff P, Sipes SL, Whyte MP. Mild hypophosphatasia mimicking severe osteogenesis imperfecta in utero: bent but not broken. Am J Med Genet 1999;86(05):434-438

16 Rodriguez E, Bober MB, Davey L, et al. Respiratory mechanics in an infant with perinatal lethal hypophosphatasia treated with human recombinant enzyme replacement therapy. Pediatr Pulmonol 2012;47(09):917-922

17 Mochizuki H, Saito M, Michigami T, et al. Severe hypercalcaemia and respiratory insufficiency associated with infantile hypophosphatasia caused by two novel mutations of the tissue-nonspecific alkaline phosphatase gene. Eur J Pediatr 2000;159(05):375-379

18 Barcia JP, Strife CF, Langman CB. Infantile hypophosphatasia: treatment options to control hypercalcemia, hypercalciuria, and chronic bone demineralization. J Pediatr 1997;130(05):825-828
19 Nakamura-Utsunomiya A, Okada S, Hara K, et al. Clinical characteristics of perinatal lethal hypophosphatasia: a report of 6 cases. Clin Pediatr Endocrinol 2010;19(01):7-13

20 Esmel-Vilomara R, Hernández S, Campos-Martorell A, GonzálezRoca E, Yeste D, Castillo F. Hypophosphatasia: novel mutation associated with an atypical newborn presentation. J Clin Res Pediatr Endocrinol 2019

21 Whyte MP. Hypophosphatasia: enzyme replacement therapy brings new opportunities and new challenges. J Bone Miner Res 2017;32(04):667-675

22 Whyte MP, Greenberg CR, Salman NJ, et al. Enzyme-replacement therapy in life-threatening hypophosphatasia. N Engl J Med 2012; 366(10):904-913

23 Whyte MP, Rockman-Greenberg C, Ozono K, et al. Asfotase alfa treatment improves survival for perinatal and infantile hypophosphatasia. J Clin Endocrinol Metab 2016;101(01): 334-342

24 Kishnani PS, Rush ET, Arundel P, et al. Monitoring guidance for patients with hypophosphatasia treated with asfotase alfa. Mol Genet Metab 2017;122(1-2):4-17

25 Gennero I, Conte-Auriol F, Salles JP. Laboratory diagnosis of hypophosphatasia. Arch Pediatr 2017;24(5S2):S57-S60 